\section{Contrast-enhanced voiding urosonography for the diagnosis of vesicoureteral reflux and intrarenal reflux: a comparison of diagnostic performance with fluoroscopic voiding cystourethrography}

Daehee $\mathrm{Kim}^{1,2}$, Young Hun Choi ${ }^{1,2}$, Gayoung Choi ${ }^{3}$, Seulbi Lee', Seunghyun Lee', Yeon Jin Cho', Seon Hee Lim ${ }^{4}$, Hee Gyung Kang ${ }^{4}$, Jung-Eun Cheon ${ }^{1,2,5}$

${ }^{1}$ Department of Radiology, Seoul National University Hospital, Seoul; ${ }^{2}$ Department of Radiology, Seoul National University College of Medicine, Seoul; ${ }^{3}$ Department of Radiology, Korea University Ansan Hospital, Ansan; ${ }^{4}$ Department of Pediatrics, Seoul National University Children's Hospital, Seoul; ${ }^{5}$ Institute of Radiation Medicine, Seoul National University Medical Research Center, Seoul, Korea

Purpose: This study evaluated the diagnostic performance of contrast-enhanced voiding urosonography (ce-VUS) using a second-generation ultrasound contrast agent for the diagnosis of vesicoureteral reflux (VUR) and intrarenal reflux (IRR), and compared it with that of standard fluoroscopic voiding cystourethrography (VCUG).

Methods: Thirty-two consecutive children from April to October 2019 were included in this study. ce-VUS and VCUG were performed simultaneously by two operators with intravesical infusion of a mixture of ultrasound contrast medium, iodinated contrast medium and water. Two pediatric radiologists independently reviewed the ce-VUS and VCUG images and reported the presence and degree of VUR (grades I-V), and the presence and type of IRR.

Results: Twenty-seven of 63 urinary systems showed VUR. Interobserver agreement for VUR grading was very good for both examinations ( $\mathrm{K}=0.87 ; 95 \%$ confidence interval $[\mathrm{Cl}], 0.82$ to 0.92 for ce-VUS and $\mathrm{K}=0.92 ; 95 \% \mathrm{Cl}, 0.87$ to 0.96 for VCUG). The detection rate of VUR showed no significant difference between the two examinations $(P=0.370)$. Four cases of VUR were missed on ce-VUS, while one case of VUR was missed on VCUG. All four false-negative cases on ce-VUS were grade 1 VUR. The two examinations showed very good agreement regarding VUR grading ( $\mathrm{K}$ $=0.89 ; 95 \% \mathrm{Cl}, 0.81$ to 0.96 ). IRR was more frequently detected with ce-VUS than with VCUG (10 cases with ce-VUS vs. 3 cases with VCUG, $P=0.016$ ).

Conclusion: ce-VUS showed very good agreement with VCUG for detecting grade 2 VUR and above, while grade 1 VUR was sometimes missed with ce-VUS. IRR was more frequently detected with ce-VUS than with VCUG.

Keywords: Vesicoureteral reflux; Ultrasonography; Contrast-enhanced ultrasonography; Intrarenal reflux; Voiding cystourethrography
ULTRA

SONO

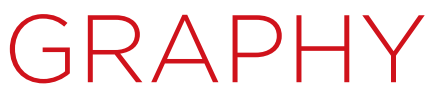

ORIGINAL ARTICLE

https://doi.org/10.14366/usg.20157 pISSN: 2288-5919 - elSSN: 2288-5943

Ultrasonography 2021;40:530-537

Received: September 28, 2020

Revised: January 25, 2021

Accepted: February 10, 2021

Correspondence to:

Young Hun Choi, MD, Department of Radiology, Seoul National University Hospital, 101 Daehak-ro, Jongno-gu, Seoul 03080, Korea

Tel. +82-2-2072-3608

Fax. +82-2-747-5781

E-mail: iater@snu.ac.kr

This is an Open Access article distributed under the terms of the Creative Commons Attribution NonCommercial License (http://creativecommons.org/ licenses/by-nc/4.0/) which permits unrestricted noncommercial use, distribution, and reproduction in any medium, provided the original work is properly cited.

Copyright (C) 2021 Korean Society of Ultrasound in Medicine (KSUM)

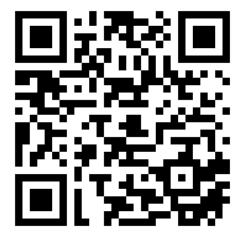

How to cite this article:

Kim D, Choi YH, Choi G, Lee S, Lee S, Cho YJ, et al. Contrast-enhanced voiding urosonography for the diagnosis of vesicoureteral reflux and intrarenal reflux: a comparison of diagnostic performance with fluoroscopic voiding cystourethrography. Ultrasonography. 2021 Oct:40(4):530-537. 


\section{Introduction}

Urinary tract infections (UTIS) are the most common bacterial infections in children [1]. About $2 \%$ of boys and $8 \%$ of girls have UTIs in the first year after their birth [2]. Recurrence of UTIs is also a common phenomenon; approximately $30 \%$ of infants with a first UTI eventually experience a recurrent UTI within 3 years [3]. Among the various risk factors for UTI, vesicoureteral reflux (VUR) is the most common among children presenting with their first UTI, and is detected in $25 \%-40 \%$ of children and $20 \%$ of neonates with their first UTI episode $[4,5]$. Over the past decades, the detection of childhood genitourinary abnormalities, including VUR, has been employed as a strategy to protect the kidneys from further damage after an initial UTI.

Voiding cystourethrography (VCUG) and radionuclide cystography (RNC) are preferred imaging modalities for VUR evaluation. VCUG has a higher radiation dose than RNC and a lower sensitivity due to intermittent imaging; however, the standardized international grading system for VCUG and its ability to depict anatomical structures in detail make it preferable for initial studies. The reflux grade determined using VCUG is the most important finding for predicting the prognosis of VUR. High-grade VUR (grade 3-5) is associated with a higher rate of recurrent UTIs and a shorter recurrence-free survival time [3]. Recurrent UTIs associated with VUR can lead to ongoing pyelonephritic damage, which, if left untreated, can cause renal failure [6]. Another important prognostic factor of VUR is the reflux involvement of the kidney's tubular system, which is known as intrarenal reflux (IRR). IRR is not a rare phenomenon in children with VUR; specifically, it has been reported in $11 \%$ of all VUR cases and up to $44 \%$ of patients with high-grade VUR [7]. IRR is known to be the main cause of renal injury and renal scarring in children with VUR. Parenchymal reflux sites have been found to show a high correlation with photon defects on dimercaptosuccinic acid (DMSA) renal scans, and can eventually become renal scars [8].

Ultrasonography (US), which is widely used in children as a noninvasive, easily accessible imaging technique, has primarily been utilized as an initial screening modality to evaluate structural abnormalities in the urinary system [9]. With the clinical introduction of several US contrast agents, contrast-enhanced US has been utilized to evaluate solid and cystic lesions of the kidney [10]. It has been also possible to evaluate VUR with US through contrastenhanced voiding urosonography (ce-VUS). Several studies have shown ce-VUS to be a valid alternative method for the assessment of VUR $[11,12]$. Furthermore, studies using a second-generation US contrast agent, SonoVue, showed ce-VUS to be comparable to VCUG in detecting and grading VUR, with good interobserver agreement $[5,13]$. While prior studies provided a parallel comparison of ce-VUS and VCUG, data were not obtained in a temporally simultaneous manner, and the detection of IRR was also not incorporated.

Therefore, the purpose of this study was to further elaborate on the potential of ce-VUS in detecting VUR and IRR, by directly comparing ce-VUS and VCUG using a mixture of US contrast medium and iodinated contrast medium.

\section{Materials and Methods}

\section{Compliance with Ethical Standards}

This retrospective study was approved by the institutional review board of Seoul National University Hospital in Korea (2008-1271149 ) and the requirement for written informed consent was waived.

\section{Study Population}

This study included all children below 18 years of age who were referred to the radiology department between April 2019 and October 2019 for the evaluation of VUR, and underwent VCUG and ce-VUS simultaneously according to the institutional protocols described below.

Reflux studies are not performed routinely after the first febrile UTI at the authors' institution. Reflux studies are generally requested for (1) children with recurrent UTIs and (2) children with their first UTI who have any suspicious findings such as hydronephrosis or scarring on baseline US that would suggest either VUR or obstructive uropathy. However, the final decision was made at the discretion of the clinician.

\section{Simultaneous VCUG and ce-VUS Techniques}

Two pediatric radiologists (Y.H.C. (A) with 10 years of experience in pediatric radiology and S.H.L. (B) with 3 years of experience, respectively) performed VCUG and ce-VUS simultaneously in the fluoroscopic room. This institutional protocol was established as a temporary protocol before ce-VUS was formally introduced and eventually replaced VCUG. The detailed protocol is as follows.

Patients were laid down on the fluoroscopic device table for the examination. The bladder was emptied before contrast mixture infusion with aseptic transurethral catheterization using a 5-8 $\mathrm{Fr}$ feeding tube. The contrast agent mixture was hung 1 meter above the examination table and was allowed to slowly infuse into the bladder by force of gravity. The contrast media mixture was composed of US contrast medium $(0.3 \mathrm{~mL}$, SonoVue, Bracco, Milan, Italy), iodinated contrast medium (40 mL, Omnipaque 300), and water $(110 \mathrm{~mL})$. Bladder capacity was calculated with a simplified formula: (age in years +2$) \times 30 \mathrm{~mL}$ for children over the age of 1 year, and (weight in kilograms) $\times 7 \mathrm{~mL}$ for infants $[14,15]$. During gravity 
infusion of the contrast media mixture, one radiologist operated fluoroscopy and the other radiologist performed a contrastenhanced US examination.

All VCUG examinations were performed using Versiflex VISTA (Hitachi, Tokyo, Japan). During the study, pulsed fluoroscopy with a frame rate of 15 frames/s was used, and fluoroscopic images were obtained using the last-image-hold technique. Radiographic spot images were only obtained at the start and end of the examination. If the patient's position needed to be changed during the examination to acquire images, the mobile $\mathrm{C}$-arm tube was rotated.

All US examinations were performed using the RS85 ultrasound system (Samsung Healthcare, Seoul, Korea). A convex CA3-10A transducer was used for every exam with the vendor-provided "ceVUS specific mode," with a low mechanical index $(0.07-0.10)$ and dual display of the subtraction contrast image and the grayscale image. Longitudinal and transverse plane images of the kidneys and bladder were alternately obtained during the filling and voiding phases with patients laying supine $[14,16]$. Meticulous examination of the bladder in the transverse view was done to detect subtle reflux (grade 1 VUR) in both distal ureters.

After the first voiding, the bladder was filled again to perform a cyclic examination. Therefore, all children underwent two cycles of examinations without exception. The operator who performed VCUG performed ce-VUS in the second cycle, and the operator who performed ce-VUS performed VCUG in the second cycle.

The average examination time for the two examination cycles was 18.6 minutes (time range, 10 to 27 minutes). The examination time was highly variable, but was generally longer in older patients due to the additional time required to fill a larger bladder volume.

The formal reports on the reflux studies were compiled by aggregating the VCUG and ce-VUS findings, and any adverse events related to contrast agent allergic reactions, catheterization, and infections were also reported in the formal reports. All examinations were performed without sedation and with antibiotic prophylaxis.

\section{Image Analysis}

In January 2020, two reviewers (Y.H.C. (A) with 10 years of experience in pediatric radiology and G.C. (C) with 1 year of experience, respectively) who were blinded to the formal reports independently reviewed ce-VUS and VCUG images recorded in the picture archiving and communication system and reported the presence and degree of VUR as grade 1-5, as well as the presence of IRR on each side.

The reflux grade of VCUG was based on the International Classification of Vesicoureteral Reflux. The reflux grade of ce-VUS was based on the system established by Darge and Troeger [17], which grades the extent of pelvicalyceal and ureteral dilation into five grades in line with the internal reflux grading system for VCUG. Additionally, the presence of IRR in VCUG and ce-VUS was reported. IRR was reported to be positive if intrarenal extension of contrast medium was seen with VCUG, and if hyperechoic microbubbles were detected in the renal parenchyma with ce-VUS.

To resolve disagreements between two reviewers, a pediatric radiologist who was not involved in the reviewing process (Y.J.C. (D) with 4 years of experience in pediatric radiology) independently reviewed discordant cases. Thereafter, the three reviewers (radiologists A, C, and D) discussed and reached a consensus. We also classified IRR into three types according to its extent. Type 1 IRR was defined as a small IRR, confined to a single medullary pyramid, which was seen as a string of tiny bubbles refluxed into the medulla with ce-VUS, and a linear or small nodular contrast filling outside the cupping of the minor calyx with VCUG. Type 2 IRR was defined as multifocal or patchy IRR involving two or more medullary pyramids, while involving less than half of the renal parenchyma. Type 3 IRR was defined as extensive IRR involving more than half of the renal parenchyma.

\section{Statistical Analysis}

Interobserver agreement was assessed with kappa analysis. The detection rates of VUR and IRR between two examinations were compared using the McNemar test. The agreement between two examinations regarding the VUR grading was evaluated using weighted kappa analysis.

All statistical analyses were performed using SPSS (version 25.0 for Windows, IBM Corp., Armonk, NY, USA). A P-value of less than 0.05 was considered to indicate statistical significance. Kappa coefficients are interpreted to indicate agreement as follows: none to slight, $0.01-0.20$; fair, $0.21-0.40$; moderate, $0.41-0.60$; substantial, $0.61-0.80$; and very good, $0.81-1.00$.

\section{Results}

In this study, a total of 32 consecutive children (20 boys and 12 girls; median age, 17 months; age range, 3 months to 16 years) underwent VUR and IRR examinations. All examinations were successfully accomplished and all 32 patients were included in this study. Among the 32 children, including one child who had undergone kidney transplantation, a total of 63 urinary systems were evaluated.

Fourteen children (27 urinary systems) showed VUR. The distribution of VUR grades was as follows: grade $1(n=6)$, grade 2 $(n=7)$, grade $3(n=9)$, grade $4(n=4)$, and grade $5(n=1)$. Anatomical abnormalities were detected in six urinary systems of five children. The abnormalities detected were as follows: multicystic dysplastic 
kidney $(n=1)$, duplex kidney with ureterocele $(n=1)$, duplex kidney without ureterocele $(n=1)$, isolated ureterocele $(n=1)$, renal agenesis with ureterocele $(n=1)$, and posterior urethral valve $(n=1)$.

No adverse events related to the examinations were reported.

\section{VUR Grading}

The detection rate of VUR was $85 \%$ (23 of 27) with ce-VUS and $96 \%$ (26 of 27) with VCUG. The detection rate of VUR was not significantly different between the two examinations $(P=0.370)$. Four cases of VUR were missed on ce-VUS, while one case of VUR was missed on VCUG. All four false-negative cases on ce-VUS were grade 1 VUR. One missed case on VCUG was grade 2 VUR (Table 1).

Table 1. Distribution of VUR grades by urinary system on ce-VUS and VCUG

\begin{tabular}{cccccccc}
\hline \multirow{2}{*}{ VUR grade } & \multicolumn{7}{c}{ VCUG } \\
\cline { 2 - 5 } & 0 & 1 & 2 & 3 & 4 & 5 & \\
\hline ce-VUS & & & & & & & \\
0 & 36 & 4 & 0 & 0 & 0 & 0 & $40(63.5)$ \\
1 & 0 & 2 & 0 & 0 & 0 & 0 & $2(3.2)$ \\
2 & 1 & 0 & 6 & 2 & 0 & 0 & $9(14.3)$ \\
3 & 0 & 0 & 1 & 6 & 1 & 0 & $8(12.7)$ \\
4 & 0 & 0 & 0 & 0 & 3 & 0 & $3(4.8)$ \\
5 & 0 & 0 & 0 & 0 & 0 & 1 & $1(1.6)$ \\
Total & $37(58.7)$ & $6(9.5)$ & $7(11.1)$ & $8(12.7)$ & $4(6.3)$ & $1(1.6)$ & 63 \\
\hline
\end{tabular}

Values are presented as numbers of affected kidneys (\%).

VUR, vesicoureteral reflux; ce-VUS, contrast-enhanced voiding urosonography; VCUG, voiding cystourethrography.
The two examinations showed very good agreement regarding VUR grading ( $\mathrm{k}=0.89 ; 95 \%$ confidence interval $[\mathrm{Cl}], 0.81$ to 0.96 ).

\section{Detection of IRR}

Ten cases of IRR were detected in 27 urinary systems with VUR. The distribution of IRR according to VUR grade was as follows: grade 1 $(n=0)$, grade $2(n=0)$, grade $3(n=6)$, grade $4(n=3)$, and grade 5 $(n=1)$. The distribution of each IRR type was as follows: type $1(n=3)$, type $2(n=6)$, and type $3(n=1)$. IRR was more frequently detected with ce-VUS than with VCUG (Figs. 1-3). While three cases $(30 \%$, 3 of 10) of IRR were detected by VCUG, 10 cases (100\%, 10 of 10), including the three cases detected by VCUG reflux, were detected with ce-VUS ( $P=0.016)$. The IRR detection rates of VCUG according to the types of IRR were $66 \%$ (2 of 3 ) for type 1 IRR, $16 \%$ (1 of 6 ) for type 2 IRR, and $0 \%$ (0 of 1 ) for type 3 IRR.

The interobserver agreement for VUR grading and IRR was very good for both examinations ( $\mathrm{K}=0.87 ; 95 \% \mathrm{Cl}, 0.82$ to 0.92 for ceVUS and $\mathrm{K}=0.92 ; 95 \% \mathrm{Cl}, 0.87$ to 0.96 for VCUG).

\section{Discussion}

Reducing the radiation dose while maintaining image quality has always been an issue with imaging modalities involving radiation, especially for young patients who are particularly susceptible to the adverse effects of ionizing radiation. The evaluation methods of choice for VUR have been VCUG and RNC. Although advances in techniques and equipment have reduced the radiation dose, exposure to radiation cannot be avoided due to the nature of these

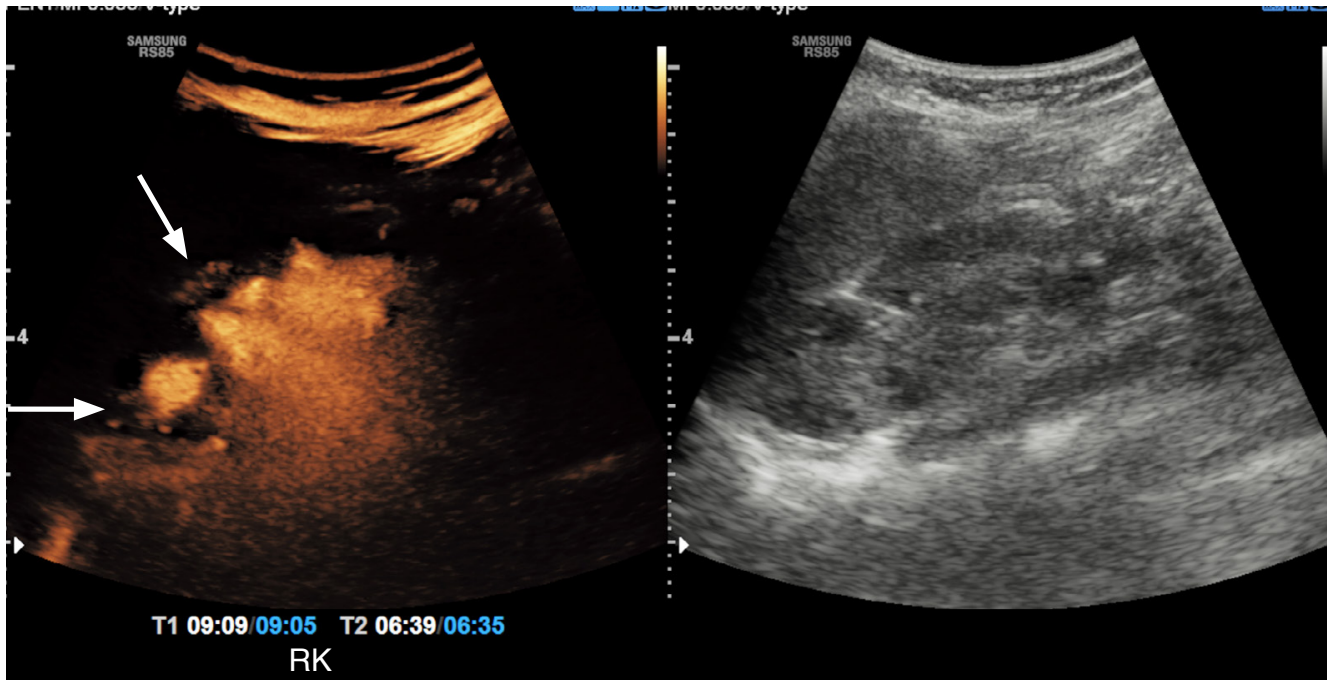

A

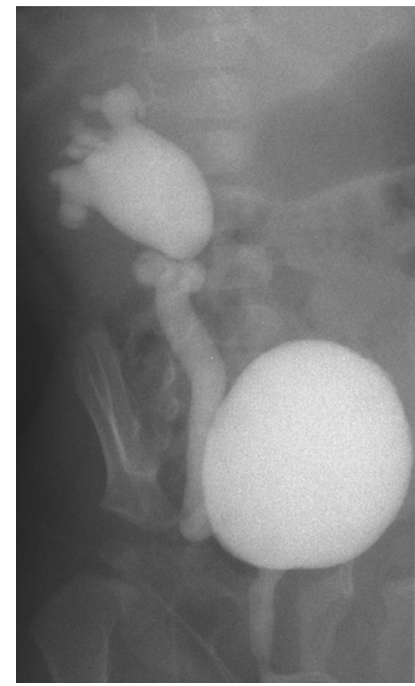

B

Fig. 1. A 1-year-old boy after a UTI with right grade 4 VUR.

A. Dual screen contrast-enhanced ultrasound image was acquired at the right kidney. Type 2 IRR is marked with arrows. B. No IRR is detected on VCUG. UTI, urinary tract infection; VUR, vesicoureteral reflux; IRR, intrarenal reflux; VCUG, voiding cystourethrography. 
studies $[9,18]$.

The use of US contrast media has allowed ce-VUS to provide functional information on reflux in addition to detailed anatomical information about the urinary system without any radiation risk [16]. No adverse effects were reported related to 4,131 intravesical applications of SonoVue in a questionnaire-based survey, and the European Federation of Societies for Ultrasound in Medicine and Biology recently approved the use of SonoVue $[19,20]$.

Therefore, ce-VUS is now advocated as a safe and radiation-free alternative for evaluating VUR in children, and several studies using SonoVue showed that ce-VUS was comparable to VCUG in detecting and grading VUR, with good interobserver agreement $[11,12]$. However, while prior studies provided parallel comparisons between ce-VUS and VCUG, we directly compared VUR detection and grading between ce-VUS and VCUG by performing the two examinations simultaneously. Considering the dynamic and intermittent nature of VUR, our study design (simultaneous performance of two examinations) would be more appropriate for a direct comparison

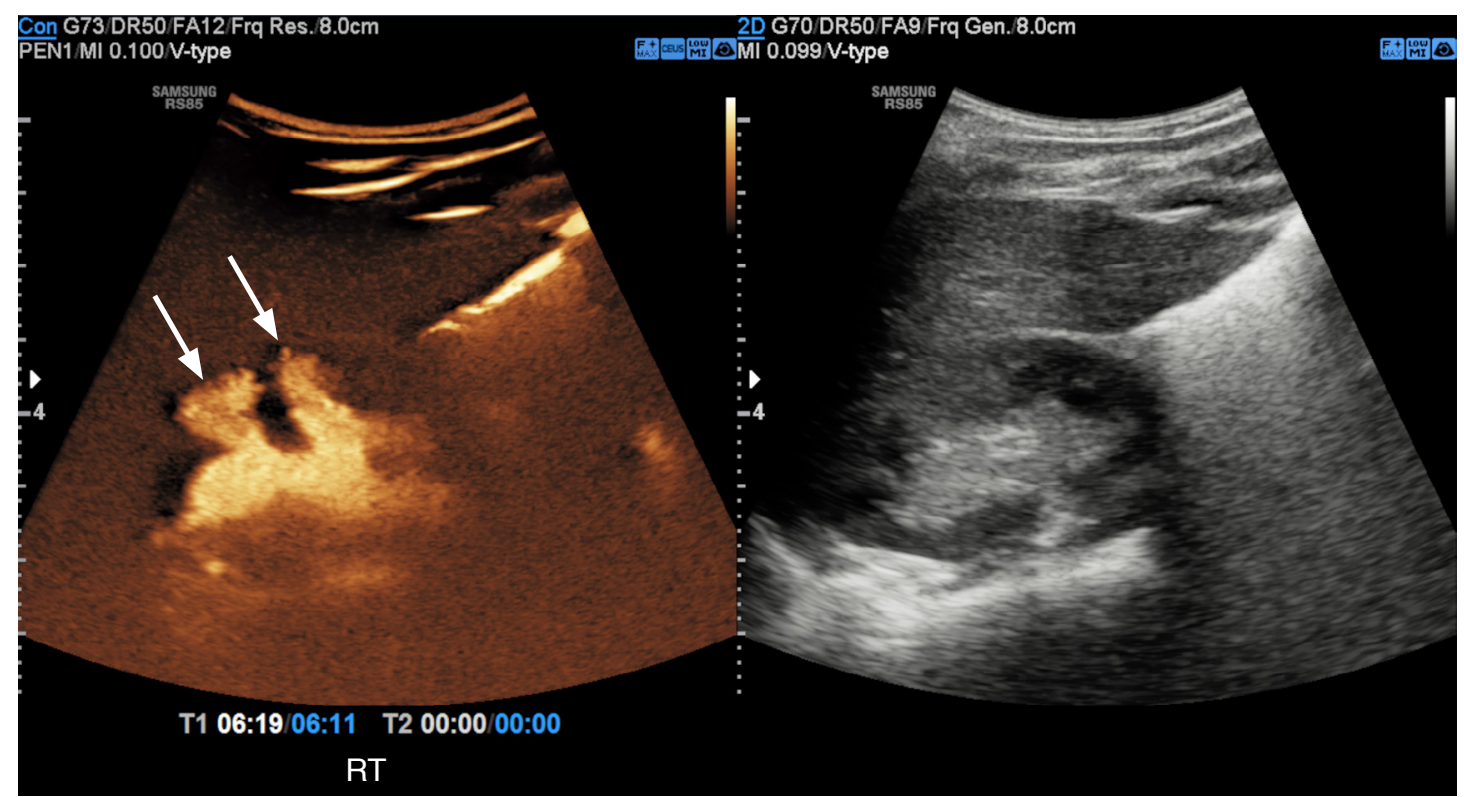

A

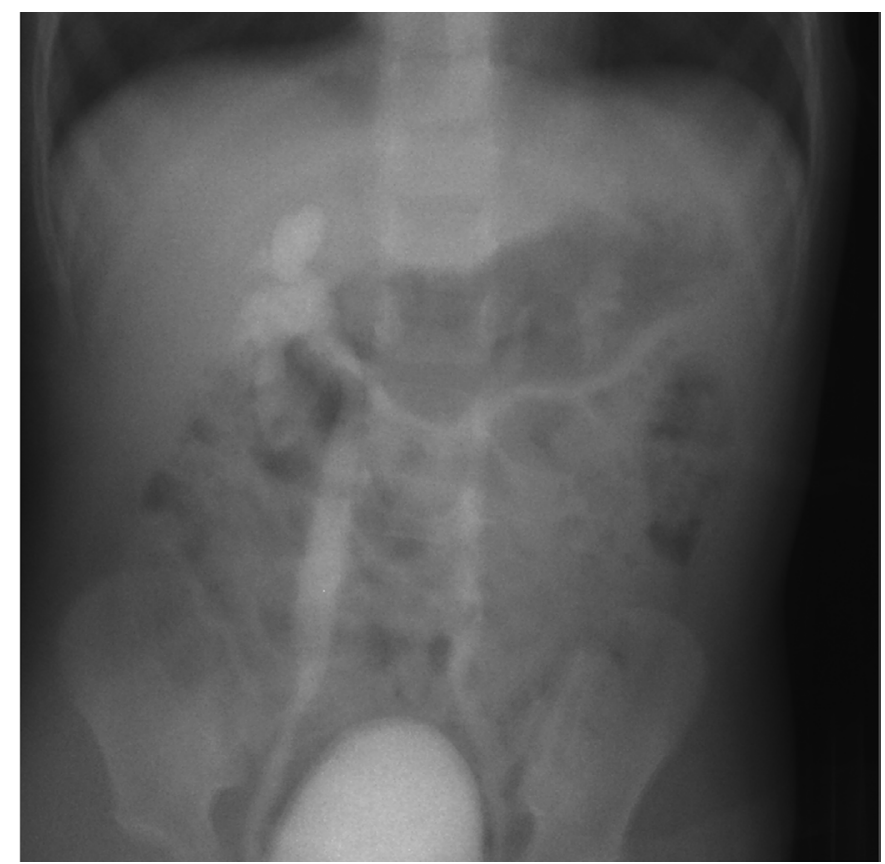

Fig. 2. A 2-year-old girl with a history of recurrent UTIs with right grade 3 VUR and left grade 2 VUR.

A. Dual screen contrast-enhanced ultrasound image was acquired at the right kidney. Type 2 IRR is marked with arrows. B. No IRR is detected on VCUG. UTI, urinary tract infection; VUR, vesicoureteral reflux; IRR, intrarenal reflux; VCUG, voiding cystourethrography. 
of diagnostic performance between VCUG and ce-VUS than the sequential performance described in prior studies.

The findings of our study are in accordance with previous studies; the detection rate of VUR using ce-VUS was comparable to that of VCUG, with very good interobserver agreement for VUR grading $[5,13]$.

However, four cases of low-grade VUR (grade 1) were missed with
ce-VUS in our study. Similar results were found in previous studies, where missed cases of reflux using ce-VUS were low-grade VUR $[5,13]$. Missed low-grade VUR cases in ce-VUS may be attributable to the characteristics of US. Unlike VCUG, ce-VUS has a limited field of view and it cannot cover the whole urinary system in a single plane, which limits its ability to detect low-grade and transient VUR. Furthermore, the sonic window of the lower urinary system is often

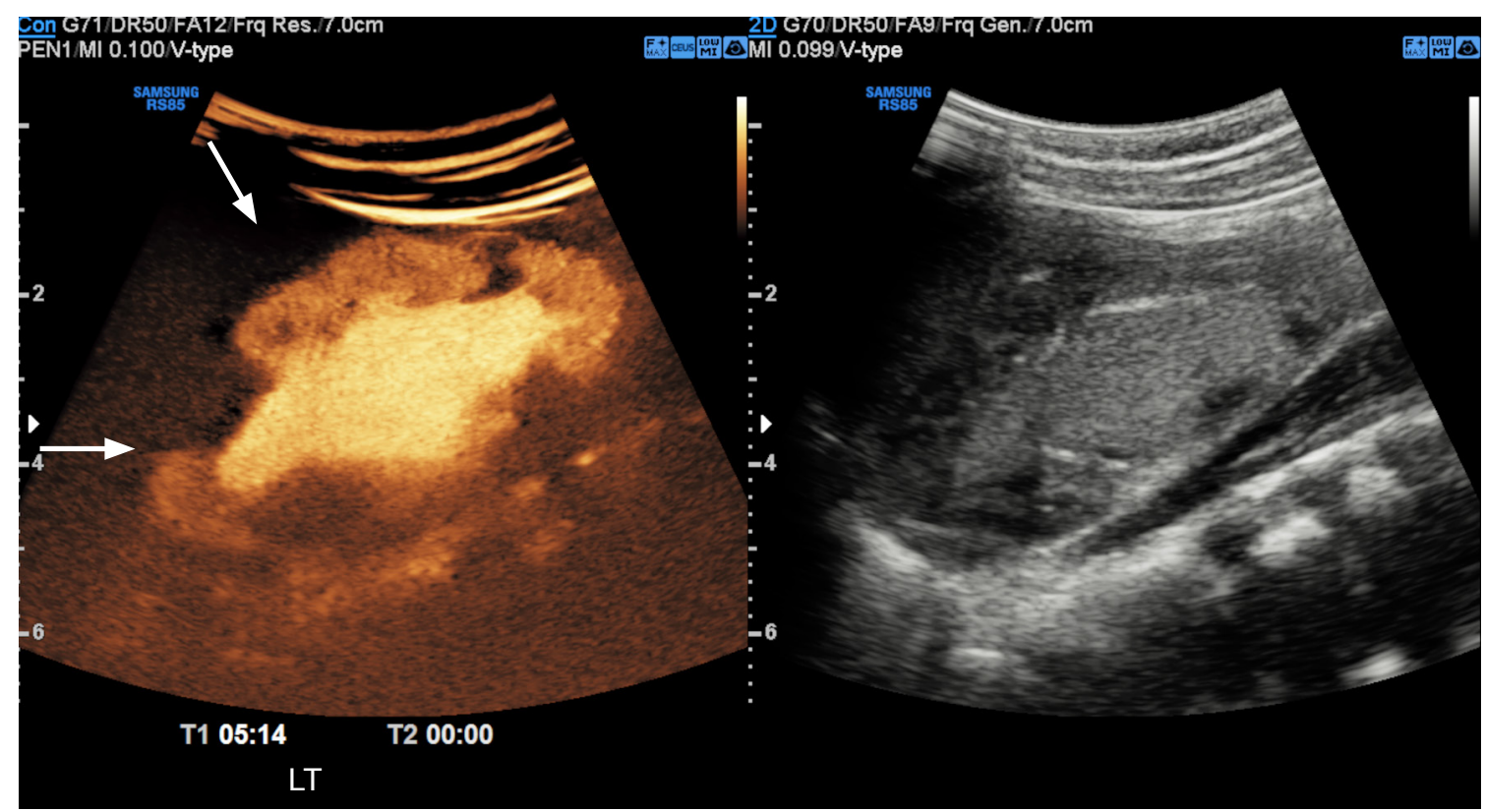

A

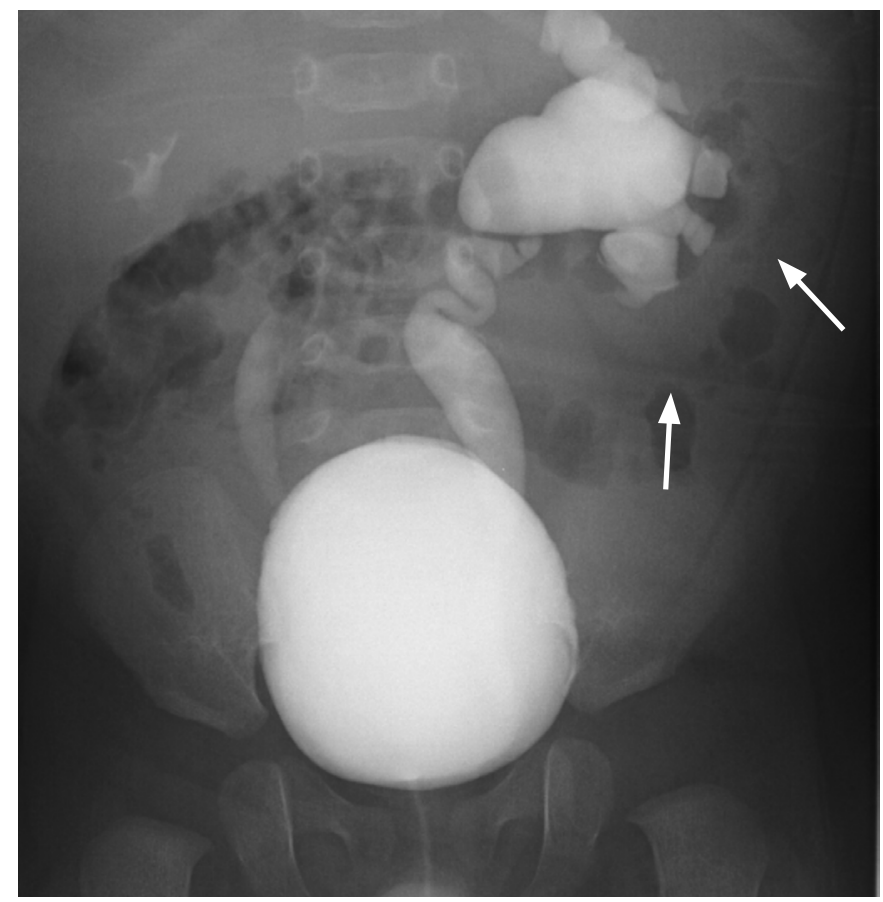

B
Fig. 3. A 3-month-old boy with a history of recurrent UTIs with left grade 5 VUR and right grade 3 VUR.

A. Dual screen contrast-enhanced ultrasound image was acquired at the left kidney. Type 3 IRR is marked with arrows. B. Reviewers reported no IRR on VCUG. However, diffusely increased renal shadow (arrows) suggests that there is extensive IRR. UTI, urinary tract infection; VUR, vesicoureteral reflux; IRR, intrarenal reflux; VCUG, voiding cystourethrography. 
limited due to overlying structures, such as gas-filled bowel loops, which further hinder the detection of low-grade reflux. Additionally, the poor image quality of B-mode US makes it more difficult to identify the distal ureters posterior to the bladder with confidence.

Fortunately, spontaneous resolution of low-grade reflux is common in low-grade VUR; it is reported in nearly $80 \%$ of cases of VUR grade 1 or 2 within 4 to 5 years of follow up. Patients with low-grade VUR without complications are recommended to receive conservative management, including active surveillance, rather than continuous antibiotic prophylaxis or more aggressive management [21-23]. Therefore, missed cases of low-grade reflux are unlikely to have a significant clinical impact on patients' management and prognosis. However, cases of low-grade VUR with febrile episodes are not clinically insignificant; furthermore, the limited number of missed VUR cases in ce-VUS cases and the lack of data on the use of only ce-VUS to detect VUR hinder a complete understanding of the clinical impact of missed cases of low-grade VUR. Further studies are needed for understanding the true extent of missed cases of low-grade VUR using ce-VUS case and their clinical impact.

One of the novel findings of this study is the advantage of ceVUS in detecting IRR. In our study, 44\% of children (14 of 32) and $43 \%$ of urinary systems (27 of 63 ) showed VUR. Among them, $48 \%$ (13 of 27) had low-grade VUR (grade 1 or 2) and 51\% (14 of 27) had high-grade VUR (grade 3 to 5). While low-grade VUR was not related with the IRR ( 0 of 13 ), $71 \%$ of high-grade VUR cases were accompanied with IRR (10 of 14). All cases of IRR were detected by ce-VUS (10 of 10), while VCUG was only capable of detecting 30\% (3 of 10) of them.

Even though prior case reports and series have suggested that ceVUS may be comparable to or better than VCUG for IRR detection, no study has compared ce-VUS and VCUG with regards to detecting IRR. To the best of the authors' knowledge, this study is the first report evaluating the performance of ce-VUS in IRR detection. The higher detection of IRR with ce-VUS was likely due to the better spatial resolution and better contrast of ce-VUS compared to VCUG. The institutional protocol for VCUG is to obtain last-image-hold or fluoro-capture images instead of obtaining radiographic spot images. Radiographic spot images are obtained only at the start and the end of the study. This technique is known to reduce the radiation dose without compromising the image quality, and is now being advocated as the standard for VCUG at many institutions $[5,7]$. However, the inevitable lower image quality of the VCUG technique likely led to a lower detection rate of IRR. Although IRR currently does not play a major role in clinical management, IRR should not be missed on imaging since it is known to be closely related with reflux nephropathy and subsequent parenchymal scarring $[7,24,25]$. Therefore, it is suggested that increased detection using ce-VUS may contribute toward deciphering the clinical value and implications of IRR without additional radiation hazard. The detection rate of IRR most likely differs by the evaluation method according to its extent and shape; specifically, more extensive IRR may be more likely to be detected by both studies, while more subtle IRR may be more easily detected using ce-VUS. However, this study was limited by the small number of detected cases of IRR due to the small number of patients enrolled, and no statistical significance was found. To clarify the impact of IRR detected with ce-VUS, further study is needed $[18,24,25]$.

To conclude, ce-VUS showed very good agreement with VCUG in detecting VUR grade 2 or above without missing high-grade VUR. IRR was more frequently detected with ce-VUS than with VCUG. Considering the similar performance of detecting and grading VUR, better detection ratio of IRR, and advantage of being a radiationfree modality, ce-VUS may have potential to be the initial modality of choice for evaluating VUR in children with UTIs.

ORCID: Daehee Kim: https://orcid.org/0000-0002-5155-4789; Young Hun Choi: https://orcid.org/0000-0002-1842-9062; Gayoung Choi: https://orcid.org/00000002-2004-5228; Seulbi Lee: https://orcid.org/0000-0002-5163-3911; Seunghyun Lee: https://orcid.org/0000-0003-1858-0640; Yeon Jin Cho: https://orcid.org/00000001-9820-3030; Seon Hee Lim: https://orcid.org/0000-0001-8327-7002; Hee Gyung Kang: https://orcid.org/0000-0001-8323-5320; Jung-Eun Cheon: https://orcid.org/00000003-1479-2064

\section{Author Contributions}

Conceptualization: Kim D, Choi YH, Choi G, Lee S, Lee S, Cho YJ, Lim SH, Kang HG, Cheon JE. Data acquisition: Choi YH, Choi G, Lee $S$ (Seunghyun Lee). Data analysis or interpretation: Kim D, Choi YH, Lee $S$ (Seulbi Lee). Drafting of the manuscript: Kim D, Choi YH, Choi G, Lee S (Seulbi Lee), Lee S (Seunghyun Lee), Cho YJ. Critical revision of the manuscript: Kim D, Choi YH, Lee S (Seulbi Lee), Cho YJ, Lim SH, Kang HG, Cheon JE. Approval of the final version of the manuscript: all authors.

\section{Conflicts of Interest}

This work was supported by a grant from Samsung Medison Co. Ltd. (Seoul, South Korea). Jung-Eun Cheon serves as Editor for the Ultrasonography, but has no role in the decision to publish this article. All remaining authors have declared no conflicts of interest.

\section{Acknowledgments}

We thank Mose Kim for technical support and consulting. 


\section{References}

1. Lamas DJ, Ingelfinger JR, Rosenbaum LS. Treatment of a 6-yearold girl with vesicoureteral reflux: polling results. N Engl J Med 2011;365:e17.

2. Blumenthal I. Vesicoureteric reflux and urinary tract infection in children. Postgrad Med J 2006;82:31-35.

3. Nuutinen $M$, Uhari $M$. Recurrence and follow-up after urinary tract infection under the age of 1 year. Pediatr Nephrol 2001;16:69-72.

4. Jacobson SH, Hansson S, Jakobsson B. Vesico-ureteric reflux: occurrence and long-term risks. Acta Paediatr Suppl 1999;88:2230.

5. Wong LS, Tse KS, Fan TW, Kwok KY, Tsang TK, Fung HS, et al. Voiding urosonography with second-generation ultrasound contrast versus micturating cystourethrography in the diagnosis of vesicoureteric reflux. Eur J Pediatr 2014;173:1095-1101.

6. Peco-Antic A. Vesicoureteral reflux and renal scarring. Srp Arh Celok Lek 2007;135:679-685.

7. Schneider KO, Lindemeyer K, Kammer B. Intrarenal reflux, an overlooked entity: retrospective analysis of 1,166 voiding cysturethrographies in children. Pediatr Radiol 2019;49:617-625.

8. Kim SW, Im YJ, Hong CH, Han SW. The clinical significance of intrarenal reflux in voiding cystourethrography (VCUG). Korean J Urol 2010;51:60-63.

9. Lim R. Vesicoureteral reflux and urinary tract infection: evolving practices and current controversies in pediatric imaging. AJR Am J Roentgenol 2009;192:1197-1208.

10. Tedesco G, Sarno A, Rizzo G, Grecchi A, Testa I, Giannotti G, et al. Clinical use of contrast-enhanced ultrasound beyond the liver: a focus on renal, splenic, and pancreatic applications. Ultrasonography 2019;38:278-288.

11. Mentzel HJ, Vogt S, Patzer L, Schubert R, John U, Misselwitz J, et al. Contrast-enhanced sonography of vesicoureterorenal reflux in children: preliminary results. AJR Am J Roentgenol 1999;173:737740.

12. Darge K. Diagnosis of vesicoureteral reflux with ultrasonography. Pediatr Nephrol 2002;17:52-60.

13. Kis E, Nyitrai A, Varkonyi I, Mattyus I, Cseprekal O, Reusz G, et al. Voiding urosonography with second-generation contrast agent versus voiding cystourethrography. Pediatr Nephrol 2010;25:2289-
2293.

14. Darge K. Voiding urosonography with ultrasound contrast agents for the diagnosis of vesicoureteric reflux in children. I. Procedure. Pediatr Radiol 2008;38:40-53.

15. Fairhurst JJ, Rubin CM, Hyde I, Freeman NV, Williams JD. Bladder capacity in infants. J Pediatr Surg 1991;26:55-57.

16. Duran C, Beltran VP, Gonzalez A, Gomez C, Riego JD. Contrastenhanced voiding urosonography for vesicoureteral reflux diagnosis in children. Radiographics 2017;37:1854-1869.

17. Darge K, Troeger J. Vesicoureteral reflux grading in contrastenhanced voiding urosonography. Eur J Radiol 2002;43:122-128.

18. Linke SY, Tsiflikas I, Herz K, Szavay P, Gatidis S, Schafer JF. Ultra lowdose VCUG in children using a modern flat detectorunit. Eur Radiol 2016;26:1678-1685.

19. Riccabona M, Vivier PH, Ntoulia A, Darge K, Avni F, Papadopoulou $F$, et al. ESPR uroradiology task force imaging recommendations in paediatric uroradiology, part VII: standardised terminology, impact of existing recommendations, and update on contrast-enhanced ultrasound of the paediatric urogenital tract. Pediatr Radiol 2014:44:1478-1484.

20. Riccabona M. Application of a second-generation US contrast agent in infants and children: a European questionnaire-based survey. Pediatr Radiol 2012;42:1471-1480.

21. Elder JS, Peters CA, Arant BS Jr, Ewalt DH, Hawtrey CE, Hurwitz RS, et al. Pediatric Vesicoureteral Reflux Guidelines Panel summary report on the management of primary vesicoureteral reflux in children. J Urol 1997;157:1846-1851.

22. Alvarez N, Alvira RD, Ruiz YG, Atuan RF, Hinojosa AS, Heras $M A$, et al. Predicting long-term renal damage in children with vesicoureteral reflux under conservative initial management: 205 cases in a tertiary referral center. Cent European J Urol 2018;71:142-147.

23. Peters CA, Skoog SJ, Arant BS Jr, Copp HL, Elder JS, Hudson RG, et al. Summary of the AUA guideline on management of primary vesicoureteral reflux in children. J Urol 2010;184:1134-1144.

24. Darge K, Trusen A, Gordjani N, Riedmiller H. Intrarenal reflux: diagnosis with contrast-enhanced harmonic US. Pediatr Radiol 2003;33:729-731.

25. Colleran GC, Barnewolt CE, Chow JS, Paltiel HJ. Intrarenal reflux: diagnosis at contrast-enhanced voiding urosonography. J Ultrasound Med 2016;35:1811-1819. 